\title{
Consequências do uso de álcool em mulheres atendidas em um Centro de Atenção Psicossocial
}

\author{
Consequences of alcohol consumption among women cared for in a Psychosocial Care Center
}

Consecuencias del consumo alcohólico en mujeres atendidas en Centro de Atención Psicosocial

Sandra Cristina Pillon ${ }^{1}$, Manoel Antônio dos Santos², Larissa Missano Florido ${ }^{3}$, Juliana Regina Cafer ${ }^{4}$, Paulo Sérgio Ferreira ${ }^{5}$, Zeyne Alves Pires Scherer ${ }^{6}$, Gisela Pires de Oliveira Marchini ${ }^{7}$

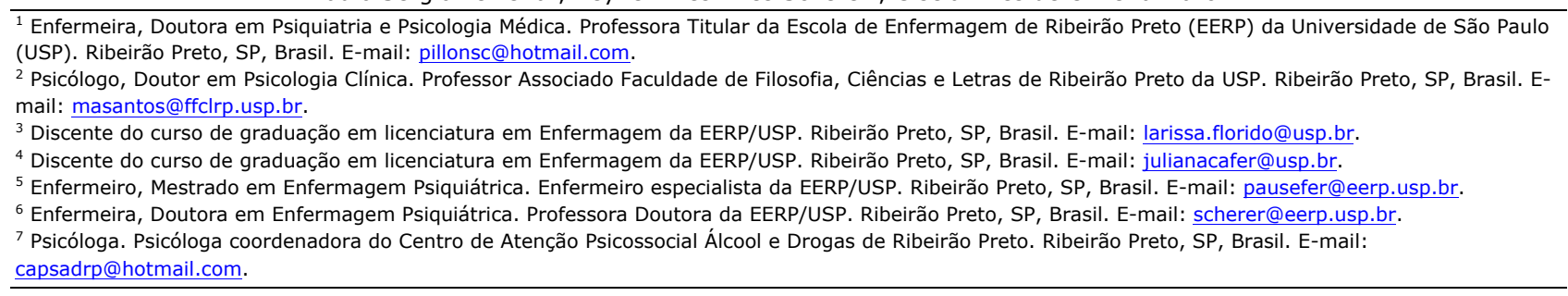

\section{RESUMO}

Este estudo teve por objetivo identificar o uso de álcool e consequências relacionadas aos aspectos físicos, interpessoais, intrapessoais, controle de impulsos e de responsabilidade social em um grupo de mulheres atendidas em um tratamento especializado. O estudo é do tipo descritivo da abordagem quantitativa. A amostra foi constituída por 61 mulheres, predominantemente adultas, solteiras, com baixo nível de escolaridade, que exerciam atividades domésticas. Em média consumiam ao dia grandes quantidades de bebidas destiladas. As mulheres apresentaram diagnóstico de síndrome de dependência do álcool com prejuízos maiores nas áreas: intrapessoal e controle de impulsos. Uma minoria buscou tratamento para parar de beber, 59\% haviam iniciado tratamento anterior por problemas mentais e psiquiátricos. Parar de beber foi a principal motivação para buscar o tratamento. Os resultados contribuem com informações sobre o consumo do álcool, bem como as consequências do beber em mulheres, essenciais para repensar o planejamento de propostas assistenciais a essa população.

Descritores: Saúde da Mulher; Transtornos Relacionados ao Uso de Álcool; Centros de Tratamento de Abuso de Substâncias; Enfermagem Psiquiátrica.

\section{ABSTRACT}

The aim of this study was to identify the consumption of alcohol and its impact on the physical, interpersonal, and intrapersonal realms, as well on the impulse control and social responsibility of a group of women receiving specialized treatment. It is a descriptive qualitative study. The sample comprised 61 women, predominately adults, single, with low education levels and who were domestic workers. On average they consumed high quantities of distilled alcohol per day. The women were diagnosed with alcohol dependence syndrome and presented significant damages to their intrapersonal and impulse control areas. A minority sought treatment to stop drinking, and $59 \%$ had initiated a prior treatment for mental and psychiatric disorders. The results provided use with information on alcohol consumption, as well as on the consequences of drinking among women. Such data is essential for rethinking how to plan health care proposals for this population.

Descriptors: Women's Health; Alcohol-Related Disorders; Substance Abuse Treatment Centers; Psychiatric Nursing.

\section{RESUMEN}

Se objetivó identificar el consumo alcohólico y las consecuencias relacionadas a aspectos físicos, interpersonales, intrapersonales, control de impulsos y de responsabilidad social en un grupo de mujeres atendidas en tratamiento especializado. Estudio descriptivo, de abordaje cuantitativo. Muestra constituida por 61 mujeres, mayoritariamente adultas, solteras, con bajo nivel de escolarización, que ejercían tareas domésticas. Consumían un alto promedio diario de bebidas destiladas. Las mujeres presentaban diagnóstico de síndrome de dependencia alcohólica con deterioros mayores en las áreas: interpersonal y control de impulsos. Una cantidad minoritaria buscó tratamiento para dejar de beber, el 59\% había iniciado tratamiento previamente por problemas mentales y psiquiátricos. Dejar la bebida constituyó el principal motivo de búsqueda de tratamiento. Los resultados proveen informaciones sobre consumo alcohólico, así como acerca de las consecuencias del alcoholismo en mujeres, esenciales para reconsiderar la planificación de propuestas de atención para tal segmento poblacional.

Descriptores: Salud de la Mujer; Trastornos Relacionados con Alcohol; Centros de Tratamento de Abuso de Substâncias; Enfermería Psiquiátrica. 


\section{INTRODUÇÃO}

Os prejuízos relacionados ao abuso de álcool situamse entre as principais ameaças para a saúde da população. O uso abusivo de álcool é considerado o maior fator de risco para morbimortalidade em pessoas com idade entre 15 e 59 anos, devido aos traumas, exposição à violência e doenças cardiovasculares. Globalmente, $6,2 \%$ de todas as mortes do sexo masculino são atribuíveis ao uso abusivo de álcool, em comparação com $1,1 \%$ dos óbitos femininos. Os homens $(7,4 \%)$ também apresentam taxas muito maiores na carga global atribuída ao álcool quando comparados às mulheres (1,4\%). Estima-se que a proporção é de quatro homens para uma mulher no consumo episódico semanal do beber pesado, o que provavelmente explica as altas taxas de morte e incapacidade ${ }^{(1)}$.

O consumo de álcool e/ou de outras drogas em mulheres tem sido crescente nas últimas décadas, constituindo um problema de saúde pública incapacitante ${ }^{(1)}$. Historicamente, os modelos de tratamento foram estruturados e embasados nas características masculinas e não contemplavam as peculiaridades do sexo feminino; como consequência, as mulheres ficaram por muito tempo subestimadas nas pesquisas $^{(2-3)}$.

A presença do consumo de álcool no sexo feminino foi identificada com crescimento linear. Em estudos epidemiológicos nacionais sobre o consumo de álcool dos brasileiros $^{(4-5)}$, as diferenças entre os gêneros foram destacadas. Os homens bebem de modo diferenciado, sendo maiores consumidores (65\%) quando comparados às mulheres $(41 \%)$. Entretanto, 0 dado mais surpreendente foi a quantidade que as mulheres consumiam: $33 \%$ bebiam em grandes quantidades (no padrão binge), ou seja, cinco ou mais doses, na vez em que mais beberam no último $\mathrm{ano}^{(4)}$. Em novo levantamento, realizado em 2012, notou-se um consumo crescente do beber regular, quando comparado aos dados de 2006; o beber em binge na mulher evoluiu de 36\% para $49 \%$, representando aumento de $36 \%$ na população feminina ${ }^{(5)}$.

Outro estudo epidemiológico mostrou um dado preocupante em relação aos índices de uso de álcool entre as mulheres das grandes cidades paulistas, com prevalência de $60 \%$ do alcoolismo ${ }^{(6)}$. Considerando o contexto internacional, a literatura estima que, dos 15,1 milhões de pessoas que fazem uso abusivo de álcool ou que são dependentes de álcool, quase um terço (aproximadamente 4,6 milhões) são mulheres e dessas, $25,4 \%$ são potenciais usuárias de serviços especializados para dependência do álcool ${ }^{(7)}$.
Apesar de ser um estudo da década de 1990, os dados indicam uma projeção do número de mulheres que necessitam de tratamento. A proporção de mulheres usuárias de substância psicoativas em tratamento tem aumentado nos últimos anos. A literatura sobre esse tema ainda é relativamente escassa. No entanto, nas últimas décadas encontra-se maior produção de estudos que evidenciam aumento na prevalência de mulheres usuárias abusivas de álcool, bem como as diferenças de gênero em relação às consequências do beber nos aspectos sociais, psicológicos e físicos ${ }^{(3)}$. Além disso, os estudos disponíveis foram conduzidos com amostras clínicas restritas, o que gera limitações metodológicas ${ }^{(2-}$ 3,8-9), sendo que há uma parcela expressiva de estudos de revisão de literatura(10-11).

O consumo de substâncias psicoativas foi incrementado pelo movimento de emancipação feminina, na medida em que as mulheres assumiram novos papéis e responsabilidades sociais, tornando-se mais competitivas no mercado de trabalho e na vida pública, o que repercutiu na reprodução de comportamentos que anteriormente eram mais comuns aos homens, como o uso do tabaco e álcool ${ }^{(3)}$. Trabalhar fora do lar e frequentar bares são exemplos de ações compartilhadas que ampliam as oportunidades femininas para consumir bebidas alcoólicas ou mesmo outras substâncias psicoativas $^{(3)}$. Por outro lado, as mulheres tendem a omitir e encobrir seu consumo, temendo represália social, o que constitui uma barreira para a busca de tratamento(10-11). Mulheres usuárias de substâncias psicoativas (ilícitas e/ou ilícitas) são estigmatizadas pelo preconceito social que lhes é imposto e permanecem no anonimato, gerando a exclusão nos sistemas de saúde e social vigentes ${ }^{(11)}$.

Os profissionais de saúde têm dificuldade de identificar os problemas relacionados ao consumo de álcool. Os problemas clínicos apresentados não são reconhecidos como sintomas secundários ao abuso de álcool $^{(6-9)}$. Por essa razão, avaliar especificamente as peculiaridades do gênero é de crucial importância no desenvolvimento de programas específicos para mulheres nos serviços especializados ${ }^{(1,11)}$.

Programas de tratamento destinados especificamente às mulheres têm se constituído em uma demanda urgente não apenas para tratar o abuso de substâncias psicoativas, como também problemas relacionados às necessidades especiais da população feminina e às barreiras enfrentadas no tratamento(10-11). Apesar de muitos serviços reconhecerem e considerarem as diferenças de gênero em usuários que buscam 
tratamento, as peculiaridades do sexo feminino têm sido ainda timidamente exploradas.

Estudo de revisão da literatura mostrou consistentes diferenças de gênero nas consequências do consumo abusivo de álcool. Especificamente, as mulheres parecem sofrer consequências negativas mais graves do consumo de álcool e de forma mais precoce e em maior grau, quando comparadas aos homens. As consequências que se assemelham, por exemplo, referem aos efeitos negativos sobre o funcionamento cognitivo e o motor, mesmo com baixas doses de álcool, o que pode desencorajar a maioria das mulheres de consumirem níveis excessivos de álcool. As consequências que mais se diferenciaram referem-se à precariedade na saúde reprodutiva, fatos que podem inibir o consumo abusivo de álcool em mulheres, resultando em menores taxas de doenças ou problemas relacionados ao uso de álcool em mulheres quando comparadas aos homens ${ }^{(12)}$.

Existem fatores ambientais específicos que contribuem para o desenvolvimento precoce do alcoolismo em mulheres, como o baixo nível socioeconômico, a presença do alcoolismo em um dos pais, experiências infantis negativas, uso da substância pelos parceiros, comportamento dos amigos em relação ao consumo de bebidas alcoólicas e baixa capacidade de controle dos impulsos frente às adversidades do meio ${ }^{(8-}$ 9).

Para melhor entendimento dos prejuízos físicos e mentais na mulher, a literatura evidencia a metabolização do álcool de maneira mais lenta. Assim, ela se torna suscetível aos prejuízos associados ao seu consumo, mesmo ingerindo níveis baixos de álcool por período de tempo mais curto(12). Apesar de consumirem quantidades menores de álcool quando comparadas aos homens, mulheres tendem a desenvolver o abuso e a dependência mais rapidamente. Trata-se do "efeito telescópio", um conceito utilizado na literatura especializada, segundo o qual as mulheres iniciam o tratamento com histórico mais curto de problemas relacionados ao uso do álcool do que os homens, mas com sintomas equivalentes ${ }^{(3)}$. Por outro lado, quantidades iguais de álcool afetam homens e mulheres de maneiras diferentes, devido às diferenças no peso e composição corporal. No entanto, os homens precisam de 45 a $50 \%$ a mais de etanol para alcançar o mesmo nível sanguíneo de álcool quando comparados às mulheres $^{(6,9)}$.

A literatura ${ }^{(9-11)}$ reforça a necessidade urgente de maiores investimentos nos serviços especializados que atendem esta população, que muitas vezes tem suas necessidades ignoradas, sendo esta situação uma das barreiras que dificultam a manutenção do tratamento de mulheres usuárias ou dependentes de álcool e/ou de outras drogas. Uma lacuna importante da literatura diz respeito à investigação das consequências psicossociais do beber em mulheres. Considerando a escassez de pesquisas sobre a temática, este estudo teve por objetivo identificar o uso de álcool e suas consequências nos aspectos físicos, interpessoais, intrapessoais, controle de impulsos e de responsabilidade social em um grupo de mulheres atendidas em um serviço especializado.

\section{MÉTODO}

Estudo descritivo exploratório de abordagem quantitativa.

O estudo foi realizado em um Centro de Atenção Psicossocial - Álcool e Drogas do interior paulista. De 70 $(100 \%)$ participantes de um grupo terapêutico específico para mulheres, em um período de três meses em 2010, foram entrevistadas 61 (87\%) mulheres que aceitaram participar da pesquisa. Foram critérios de inclusão: participar do grupo psicoterapêutico para mulheres, oferecido pelo referido serviço. Como critérios de exclusão estabeleceram-se: mulheres em estado de embriaguez ou uso prévio de drogas, sintomas psicóticos produtivos, prejuízos cognitivos severos, avaliados pela Escala Breve de Avaliação Psiquiátrica - BPRS(13). Ao avaliar os critérios de exclusão por meio dessa escala, a pontuação variou de zero a 16 pontos, sendo a moda dois pontos. Assim, todas as pessoas convidadas apresentaram condições satisfatórias para participar do estudo.

É importante mencionar que diante do convite aos participantes para realizar a entrevista na presente pesquisa, não houve recusas.

A coleta de dados foi realizada por meio de entrevista individual, aplicada por pesquisadores devidamente treinados, em sala reservada do serviço, após o término das sessões de psicoterapia de grupo, respeitando os princípios éticos em pesquisa clínica.

Para a coleta de dados utilizou-se um questionário contendo informações sociodemográficas, padrão de consumo do álcool e tipo de droga utilizada, baseando-se no tipo de substância, quantidade, frequência e os problemas e consequências negativas do comportamento do beber por meio do The Drinker Inventory of Consequences (DrInC) ${ }^{(14-15)}$.

A DrInC é um inventário dividido em cinco domínios: (1) Consequências físicas (8 itens): refere-se às diversas 
consequências do beber abusivo, incluindo os efeitos agudos e crônicos da embriaguez; os itens avaliam: ressaca, problemas do sono, doenças, prejuízos à saúde, hábito alimentar e danos relacionados ao beber. (2) Consequências interpessoais (10 itens): enfocam os impactos do beber sobre as relações interpessoais; as consequências adversas incluem: prejuízos ou perdas de amizades, relacionamento amoroso ou familiares em função do beber, danos à reputação e ações cruéis ou confusas quando embriagada. (3) Consequências intrapessoais (8 itens): referem-se às percepções subjetivas, que podem ser observáveis por outros; incluem sentimentos ruins, infelicidade por causa da bebedeira, experiências de mudanças de personalidade para pior, interferências no crescimento pessoal e na vida moral/espiritual, atividades e interesses, levar a vida do jeito que quer. (4) Consequência na responsabilidade social ( 7 itens): enfocam as repercussões no cumprimento de papéis sociais, observadas por outros; incluem problemas no trabalho e/ou escola (ausência, qualidade empobrecida no trabalho, suspensão ou demissão do emprego), problemas financeiros, envolvimento em outros problemas, falhas para atender as expectativas sociais. (5) Consequências no controle dos impulsos (12 itens): incluem as sequelas da embriaguez, uso abusivo de outras substâncias (tabaco, drogas), ações impulsivas e comportamentos de risco, brigas físicas, acidentes de trânsito após o beber e problemas com a lei, causar danos a outros ou a propriedade alheia.

A pontuação total da DrInC é o somatório dos escores, primeiro de cada domínio e, posteriormente, da escala total. As respostas são dicotômicas: Sim e Não. O escore total é o somatório dos escores de todas as subescalas. A classificação para mulheres é distribuída em uma escala de zero a 100 , classificada em: muito baixa (0-23 pontos), baixa (24-28 pontos), média (29-34 pontos), alta (35-38 pontos) e muito alta (39-45 pontos) $)^{(14)}$.

Em relação à presença de transtornos mentais, considerou-se o diagnóstico mais recente registrado no prontuário do serviço.

Para a análise estatística elaborou-se um banco de dados no programa Statistical Package Social Science (SPSS) versão 19 for Windows. Os resultados estão apresentados em número, porcentagem, média e desviopadrão.

O estudo seguiu as normas e procedimentos éticos de acordo com a Resolução 466/2012, foi aprovado pelo Comitê de Ética em Pesquisa do Centro de Saúde-Escola da Faculdade de Medicina de Ribeirão Preto, Universidade de São Paulo. Processo No 408/2010.

\section{RESULTADOS}

A amostra caracterizou-se por mulheres predominantemente adultas, com média de idade de $41,4$ anos ( $D p \pm 11,1)$, variando entre 18 e 72 anos, 22 $(36,1 \%)$ solteiras, com baixo nível de escolaridade. A respeito do trabalho, $41(67,2 \%)$ exerciam atividades domésticas e $34(55,7 \%)$ moravam em casa ou apartamento próprio, conforme apresenta a Tabela 1.

Em relação ao diagnóstico, todas as mulheres apresentaram critérios para síndrome de dependência do álcool, segundo as descrições dos prontuários do serviço. Quanto ao padrão de consumo, as mulheres bebiam ao dia, em média, 444,25 $\mathrm{ml}$ de bebidas alcoólicas destiladas $46(75,4 \%)$ e fermentadas 20 (24,5\%). O tempo de consumo dessa quantidade foi, em média, de 14 meses. Por fim, as mulheres começaram a beber em média aos 25 anos, conforme informações descritas na Tabela 2.

Tabela 1: Características sociodemográficas das mulheres participantes do grupo terapêutico $(n=61)$. Ribeirão Preto, SP, Brasil, 2010.

\begin{tabular}{|c|c|c|c|}
\hline & & $\mathbf{N}$ & $\%$ \\
\hline \multirow{4}{*}{ Estado civil } & Solteira & 22 & 36,1 \\
\hline & Casada/União estável & 17 & 27,9 \\
\hline & Divorciada & 17 & 27,9 \\
\hline & Viúva & 5 & 8,2 \\
\hline \multirow{4}{*}{ Escolaridade } & Ensino Fundamental Completo/ Incompleto & 52 & 85,2 \\
\hline & Ensino Médio Completo / Incompleto & 5 & 8,1 \\
\hline & Ensino Superior Completo / Incompleto & 1 & 1,6 \\
\hline & Não frequentou a escola & 3 & 4,9 \\
\hline \multirow{3}{*}{ Trabalho } & Trabalhos domésticos & 41 & 67,2 \\
\hline & Trabalho formal & 20 & 32,8 \\
\hline & Casa ou apartamento próprio & 34 & 55,7 \\
\hline \multirow[t]{2}{*}{ Onde mora } & Casa ou apartamento dos pais ou familiares & 14 & 22,9 \\
\hline & Outros (rua, pensão, hotel, sozinho em casa alugada) & 13 & 21,3 \\
\hline
\end{tabular}


Tabela 2: Valores mínimo, máximo, média e desvio-padrão do consumo de bebidas alcoólicas em mulheres atendidas em um grupo terapêutico ( $N=61)$. Ribeirão Preto, SP, Brasil, 2010.

\begin{tabular}{|c|c|c|c|c|}
\hline & Min & Max & Média & Dp \\
\hline Quantidade de bebida alcoólica consumida ao dia (ml/dia) & 100 & 975 & 444,2 & 34,3 \\
\hline Tempo em que bebe nessa frequência (em meses) & 1 & 30 & 14 & 4,9 \\
\hline Idade de início do beber (em anos) & 15 & 60 & 25 & 8,1 \\
\hline
\end{tabular}

Quanto à última vez em que consumiram bebidas alcoólicas, $58(95,1 \%)$ mulheres fizeram uso de álcool nas últimas 24 horas. Em relação às consequências negativas do beber, avaliadas na escala DrInc, as mulheres apresentaram prejuízos em todas os aspectos avaliados, com maior evidência nas áreas intrapessoal e de controle dos impulsos. O escore total da escala foi de 30,5 pontos (Tabela 3).

Das motivações para a busca do tratamento, parar de usar bebidas alcoólicas foi a mais respondida, 22 (36\%), conforme mostra a Tabela 4.

Tabela 3. Apresentação dos valores de média e desvio-padrão dos domínios da Escala DrInC ( $\mathrm{N}=61)$. Ribeirão Preto, SP, Brasil, 2010.

\begin{tabular}{ccc}
\hline & Média & Desvio padrão \\
\hline Intrapessoal & 7,33 & 1,89 \\
Controle de impulsos & 6,84 & 1,25 \\
Interpessoal & 5,80 & 2,08 \\
Esfera física & 5,55 & 2,56 \\
Responsabilidade social & 4,92 & 1,54 \\
Escore total da DrInC & 30,5 & 7,9 \\
\hline
\end{tabular}

Tabela 4. Motivações para a busca do tratamento referidas por mulheres participantes do grupo terapêutico ( $n=61)$. Ribeirão Preto, SP, Brasil, 2010.

\begin{tabular}{ccc}
\hline & $\mathbf{N}$ & $\mathbf{\%}$ \\
\hline Parar com o uso de bebidas alcoólicas & 22 & 36,0 \\
Encaminhamento pelos serviços de saúde & 17 & 27,8 \\
Problemas de saúde e/ou uso do álcool & 10 & 16,3 \\
Mudança de comportamento e/ou de vida & 6 & 9,8 \\
Incentivo dos familiares e/ou problemas na família & 5 & 8,1 \\
Incentivo dos amigos & 1 & 1,6 \\
\hline
\end{tabular}

Quanto ao tratamento, 36 (59\%) mulheres já haviam tentado deixar de beber, antes de iniciar o acompanhamento no serviço especializado. Dentre os motivos alegados figuram: tratamento para os problemas dos nervos, mentais, psiquiátricos ou psicológicos $29(47,5 \%)$, dados não apresentados em tabela.

\section{DISCUSSÃo}

A amostra de mulheres em tratamento no serviço especializado foi constituída predominantemente por pacientes adultas, solteiras, com baixo nível de escolaridade, que exerciam atividades domésticas (Tabela 1). Esses dados corroboram a literatura, que apresenta perfis sociodemográficos semelhantes ${ }^{(2,8)}$. Além disso, os estudos destacam baixa condição socioeconômica, quando são avaliadas mulheres em atendimento especializado para dependência de álcool ${ }^{(2,8-}$ 9), evidenciando que tal condição é muito semelhante a de homens em tratamento. Reconhece-se que gênero e idade apresentam efeitos significantes sobre o padrão do beber $^{(1-2)}$. Os dados sugerem progressão contínua do beber em binge na população feminina, o que pode ser um caminho facilitador para o desenvolvimento da dependência(1-2).

Em relação à quantidade, frequência e tipo de uso do álcool, os dados do presente estudo mostram que as mulheres consumiram bebidas de alto teor alcoólico e em grande quantidade no último ano, principalmente os destilados. Por ser um grupo de mulheres diagnosticadas com síndrome de dependência alcoólica, este consumo reflete a presença de sintomas da própria dependência. Do ponto de vista biológico, deve-se considerar o processo da metabolização do álcool, uma vez que mulheres têm um volume corporal de água menor do que o homem, o que contribui para maior concentração de álcool no sangue, expondo-as a diversos problemas de comportamento, bem como de saúde ${ }^{(3,16)}$.

Além disso, fatores sociais e culturais exercem forte influência sobre o padrão e grau de ingestão de álcool pelas mulheres, o que altera sua vulnerabilidade ao desenvolvimento de problemas decorrentes do consumo ${ }^{(16)}$. 
Quanto à idade de início do beber, a literatura descreve que quanto mais cedo se inicia o consumo, provavelmente o adoecimento decorrente do uso crônico do álcool ocorrerá de forma precoce e em maior nível de gravidade. Considerando que as mulheres do presente estudo são adultas, em torno da quarta década de vida, e que iniciaram o beber ainda bem jovens (Tabela 2), os dados obtidos corroboram a literatura ${ }^{(2-3)}$.

O início precoce do uso do álcool torna as mulheres mais vulneráveis ao desenvolvimento de diversos problemas de saúde e sociais, dando origem a determinadas preocupações clínicas e de saúde pública ${ }^{(2-}$ ${ }^{3,17)}$. Confirma-se, assim, o efeito telescópico, ou seja, mesmo com poucos anos de uso de substâncias psicoativas $^{(3)}$, as mulheres geralmente apresentam mais complicações físicas, psiquiátricas e sociais consequentes ao uso de álcool, quando comparadas aos homens ${ }^{(12)}$.

Entre as pessoas em tratamento para abuso de substâncias psicoativas, a coocorrência de sofrimento psíquico ou transtornos mentais tem sido associada a declínio do funcionamento social, bem como à qualidade de vida e de saúde geral empobrecida ${ }^{(17-18)}$.

De acordo com a literatura, a idade de início do uso, o padrão de consumo de álcool e ter recebido tratamentos anteriores são considerados fortes preditores para a entrada e manutenção no tratamento ${ }^{(2-}$ $3,9-10,17)$. Por conseguinte, os profissionais de saúde devem estar atentos para intervir de modo efetivo e precoce junto a essa população(11,20).

Dentre as consequências negativas do beber avaliadas pela escala DrInC (Tabela 3), as mulheres do presente estudo apresentaram prejuízos em todas as áreas avaliadas. Os maiores prejuízos identificados foram nas áreas: intrapessoal e controle dos impulsos. 0 escore global da escala foi de 30,5 pontos, o que caracteriza um valor elevado. As relações intrapessoais e interpessoais estão entre os principais fatores de risco para o consumo abusivo de álcool em mulheres, embora não muito diferentes dos homens ${ }^{(15,18)}$. Pesquisas sugerem que mulheres são mais suscetíveis do que os homens aos efeitos das substâncias psicoativas, relacionados a dificuldades interpessoais, traumas e consequências médicas, aumentando os riscos de morbimortalidade ${ }^{(6,16,19)}$.

Mulheres que buscam tratamento para abuso ou dependência de álcool e/ou de drogas apresentam um conjunto diversificado de problemas físicos, psicológicos e sociais complexos, incluindo transtornos mentais e sofrimento psíquico, altos índices de histórias traumáticas e problemas interpessoais, exposição a situações de violência, baixo nível de qualificação profissional e de desenvolvimento de habilidades vocacionais, baixa renda e provável dependência de álcool em níveis mais graves ${ }^{(9,16-17)}$.

Vale mencionar que a literatura sobre esse tema ainda é limitada, o que dificulta uma discussão mais profunda. Há escassez de estudos que utilizaram de forma sistemática a escala DriC em mulheres, em comparação com a utilização em amostras masculinas $^{(15)}$.

Outra limitação do presente estudo refere-se ao fato de que não foi identificado o uso do álcool pelo parceiro. Por outro lado, a amostra caracteriza-se por um grupo considerável de mulheres solteiras, divorciadas e viúvas. Assim, há necessidade de outros estudos para avaliar essa especificidade. Convém recordar que a literatura aponta de que existe uma probabilidade muito grande de mulheres alcoolistas vincularem-se a homens alcoolistas. Mas, evidencia-se também que, quando apenas um dos cônjuges apresenta problemas relacionados ao uso de álcool, as possibilidades de discórdias e conflitos são maiores, o que muitas vezes resulta em separação conjugal $^{(7,12,15-21)}$.

Dentre as principais motivações para a busca do tratamento relatadas pelas participantes, parar de beber, encaminhamento pelos serviços de saúde e problemas de saúde e/ou decorrentes do uso de álcool constituíram $80 \%$ das respostas obtidas (Tabela 4). De acordo com a literatura, a maioria dos usuários de álcool e/ou de drogas que procura ou é encaminhada para tratamento encontra-se extremamente ambivalente quanto à cessação do consumo. Ao mesmo tempo em que manifestam desejo de parar de beber, pois o uso de álcool está causando problemas, relatam desejo de continuar bebendo por prazer. Desse modo, a ambivalência é um conflito psicológico que precisa ser abordado e superado durante o tratamento ${ }^{(15,22)}$.

$\mathrm{Na}$ amostra investigada, independentemente do nível de gravidade, todas as mulheres apresentaram diagnóstico de síndrome de dependência do álcool. Nesse sentido há que considerar que os sintomas físicos e psicológicos são evidentes e que essas mulheres seriam potenciais usuárias de serviços de saúde em função das consequências decorrentes do consumo crônico do álcool ${ }^{(21)}$.

A literatura(17-21) evidencia que as complicações físicas, psíquicas e sociais figuram entre as principais razões para que alcoolistas busquem alguma forma de tratamento e, posteriormente, usem esses motivos para mudar seus comportamentos em relação ao beber. 


\section{CONCLUSÃO}

O presente estudo permitiu identificar questões peculiares sobre o consumo de álcool e as consequências negativas do beber em mulheres em tratamento para dependência atendidas em um serviço especializado.

A dependência de álcool em mulheres é um grave problema de saúde pública incapacitante e tem sido considerada um tema complexo e multifatorial. Os resultados do presente estudo contribuem para repensar as práticas assistenciais oferecidas às mulheres em tratamento em serviço especializado. O conhecimento sistematizado é crucial para auxiliar o desenvolvimento de políticas públicas direcionadas à saúde da mulher nos diferentes níveis de complexidade da atenção em saúde. As peculiaridades e especificidades do consumo de álcool em mulheres devem ser consideradas na tomada de decisão dos gestores.

Este estudo é pioneiro na utilização da escala DrInC em mulheres em atendimento em serviço especializado

\section{REFERÊNCIAS}

1. World Health Organization. Global status report on alcohol and health. WHO Genebra. 2011.

2. Elbreder MF, Laranjeira R, Siqueira MM, Barbosa DA. Perfil de mulheres usuárias de álcool em ambulatório especializado em dependência química. J Bras. Psiquiatr. 2008;57(1):9-15. 3. Wolle CC, Sanches M, Zilberman ML, Caetano R, Zaleski M, Laranjeira $R R$, et al. Differences in drinking patterns between men and women in Brazil. Rev. Bras. Psiquiatr. 2011;(33):36773.

4. Pinsky I, Zaleski M, Laranjeira R, Caetano R. Primeiro levantamento nacional sobre os padrões de consumo de álcool na população brasileira. Rev. Bras. Psiquiatr. 2010; 32(3):214-15. 5. Laranjeira R, Madruga CS, Pinsky I, Caetano R, Ribeiro M, Mitsuhiro S. II Levantamento Nacional de Álcool e Drogas. Consumo de Álcool no Brasil: Tendências entre 2006/2012. São Paulo: INPAD; 2013 [acesso em 2 março 2014]. Disponível em: http://inpad.org.br/wpcontent/uploads/2013/04/LENAD_ALCOOL_ResultadosPreliminares.pdf.

6. Prado JA, Kerr-Corrêa F, Lima MCP, Silva GGAS, Santo JLF. Relations between depression, alcohol and gender in the metropolitan region of São Paulo, Brazil. Ciênc. saúde coletiva. 2012;17(9):2425-34

7. Williams GD, Grant BF, Harford TC, Noble BA. Population projections using DSM-III criteria: Alcohol abuse and dependence, 1990-2000. Alcohol Health Res World. 1989;13(4):366-70.

8. Elbreder MF, Souza e Silva R, Pillon SC, Laranjeira R. Alcohol dependence: Analysis of factors associated with retention of patients in outpatient treatment. Alcohol Alcoholism. [Internet]. 2010 [acesso em: 10 Marc 2014]; 1-3. Disponível em: http://dx.doi.org/093/alcalc/agq078.

9. Esper LH, Corradi-Webster CM, Carvalho AMP, Furtado EF. Mulheres em tratamento ambulatorial por abuso de álcool: características sociodemográficas e clínicas. Rev Gaúcha Enferm. 2013;34(2):93-101.

10. Greenfield SF, Brooks AJ, Gordon SM, Green CA, Kropp F, McHugh RK., et al. Substance abuse treatment entry, retention, and outcome in women: A review of the literature. Drug Alcohol Depen. 2007;86,1-21. no tratamento da dependência química. Acredita-se que o enfermeiro, desde que convenientemente treinado, pode ser um profissional-chave no cenário do tratamento ao se apropriar desta escala para buscar evidências que apoiem sua prática clínica.

Frente à escassez de estudo sobre o tema, os resultados obtidos fornecem subsídios para 0 desenvolvimento futuro de estratégias de prevenção e promoção de saúde em mulheres. Nessa direção, estudos prospectivos e longitudinais são necessários para avaliar a efetividade dos programas implementados, cuja meta é reduzir os problemas decorrentes do uso de álcool.

\section{AGRADECIMENTOS:}

Fundação de Amparo à Pesquisa (FAPESP), Bolsa Treinamento Técnico - TT-1 2010/2011 e Conselho Nacional de Desenvolvimento Científico e Tecnológico (CNPq), Bolsa PIBIC 2010/2011.

11. United Nations Office on Drugs and Crime (UNODC). Substance abuse treatment and care for women: Case studies and lessons learned. 2004. Viena.

12. Clemens SL, Grant BM, Matthews. A review of the impacts of health and health behaviors on woman's alcohol use. Am J Health Behav. 2009;33(4):400-15.

13. Crippa JAS, Hallak JEC, Sanches RF, Loureiro SR, Zuardi A W. Roteiro de entrevista estruturada para a escala breve de avaliação psiquiátrica (SIG-BPRS). In: Gorestein C; Andrade LHSG, Zuardi A, ed. Escalas de avaliação clínica em psiquiatria e psicofarmacologia. São Paulo: Lemos Editorial, 2000, p.207-17. 14. Miller WR, Tonigan JS, Longabaugh R. The Drinker Inventory of Consequences (DrInc): an instrument for assessing adverse consequences of alcohol abuse. National Institute on Alcohol Abuse and Alcoholism \& Project MATCH Monograph Series 1995; 4:94p.

15. Sales CMB, Figlie NB. Revisão de literatura sobre a aplicação da entrevista motivacional breve em usuários nocivos e dependentes de álcool. Psicol. estud. 2009;14(2):333-40. 16. Edwards G, Marshall J, Cook CH. O tratamento do alcoolismo: um guia para profissionais de saúde. Porto Alegre: Artes Médicas, 4 ed., cap. 17 p. $234-48,2005$

17. Johnson PB, Richter L, Kleber HD, McLellan AT, Carise D. Telescoping of drinking-related behaviors: gender, racial/ethnic, and age comparisons. Subs Use Misuse. 2005;40(8):1139-51. 18. Nolen-Hoeksema S, Hilt L. Possible contributors to the gender differences in alcohol use and problems. J Gen Psychol. 2006;133(4):357-74.

19. Kerr-Corrêa F, Igami TZ, Hiroce V, Tucci AM. Patterns of alcohol use between genders: A cross-cultural evaluation. J Affect Disord. 2007;102,(1-3):265-75.

20. Freitas GL, Vasconcelos CTM, Moura ERF, Pinheiro AKB. Discutindo a política de atenção à saúde da mulher no contexto da promoção da saúde. Rev. Eletr. Enf. 2009;11(2):424-8. Available from: http://www.fen.ufg.br/revista/v11/n2/v11n2a26.htm. 21. World Health Organization [Internet]. Health systems performance assessment 2009 [cited 2013 Aug 9]. Available from: http://www3.who.int/cidi/.

22. Lundahl B, Moleni T, Burke BL, Butters R, Tollefson D, Butler $C$, Rollnick S. Motivational interviewing in medical care settings: 
a systematic review and meta-analysis of randomized controlled trials. Patient Educ Couns. 2013;93(2):157-68.

Artigo recebido em 21/02/2013.

Aprovado para publicação em 27/02/2014.

Artigo publicado em 30/06/2014. 